\title{
Might expert knowledge improve econometric real estate mass appraisal?
}

\author{
Mariusz Doszyń ${ }^{1}$ (D)
}

Accepted: 3 February 2022

(c) The Author(s) 2022

\begin{abstract}
The article examines whether expert knowledge improves the estimation results of real estate mass appraisal models. Six econometric models were compared: OLS, mixed, the Bayesian model, the Inequality Restricted Least Squares (IRLS) model, ridge and LASSO regression (with regularization). In three of the models (mixed, Bayesian, and IRLS) prior knowledge was applied. In mixed and Bayesian models priors took the form of intervals for model parameters. In IRLS, restrictions in the form of inequalities were applied. In the empirical example mass appraisal models were applied in the valuation of undeveloped land for residential purposes. Models with prior knowledge turned out to be the best with regard to the consistency of estimates with theory. Also, prediction accuracy was better for models with prior knowledge. In the case of low quality data expert knowledge might significantly improve estimation results of real estate mass appraisal econometric models.
\end{abstract}

Keywords Econometric models of real estate mass appraisal - Expert knowledge · Inequality Restricted Least Squares · Mixed estimation · Bayesian regression · Ridge regression · LASSO

\section{Introduction}

Information about real estate value is a very important factor that influences the efficiency of every real estate market. Knowledge about real estate value is necessary for almost all kinds of decisions made by customers, commercial and public institutions, governments. Because the number of properties is high, mass appraisals might be necessary. Mass appraisals may be needed for tax purposes, mortgage banks, estimating the economic effects of changes to spatial development plans or updating real estate fees.

Mariusz Doszyń

mariusz.doszyn@usz.edu.pl

1 Institute of Economics and Finance, Department of Econometrics and Statistics, University of Szczecin, Szczecin, Poland 
Many kinds of statistical and econometric models are used in real estate mass appraisal. In this article, it is stated that methods applied in mass valuation should depend on the quality of databases from local markets. Real estate information is often incomplete and uncertain. Real estate features such as transport availability or neighborhood are qualitative variables; hence, they are prone to subjectivity. Moreover, the variability of real estate features is often very low (e.g., neighborhoods of the same type).

Currently, machine learning (or data science) methods are very popular. For these kinds of methods, massive datasets are needed, and information quality must be satisfactory to obtain sensible results. If these conditions are fulfilled, machine learning methods might be helpful. High-quality databases are usually accessible for developed, "big" real estate markets, e.g., housing markets in large cities.

However, there are many local small markets for which it is impossible to obtain massive datasets. Land markets are an example. It is often very difficult to obtain massive datasets for undeveloped markets that are also small and inefficient. Even very sophisticated methods are useless if the information quality is low, and thus, there is no need to search for more advanced methods for low-quality databases. In this article, it is stated that in such cases, expert knowledge might increase model accuracy. In addition, real estate experts often have prior information that might improve estimation results. For example, appraisers frequently have knowledge about the impact of real estate features in the form of weights given to comparable properties.

In this article, three kinds of econometric models with prior information are considered: mixed, Bayesian and IRLS (Inequality Restricted Least Squares). In these models, prior knowledge was used in the form of restrictions or intervals for model parameters. Intervals were provided by real estate appraisers, who "knew" the local real estate market. Models with prior information were compared with OLS, ridge and LASSO regression (with regularization). OLS models are often used as a benchmark. Ridge regression and LASSO might increase estimation efficiency, especially for properly specified "shrinkage" parameters.

The article examines whether econometric real estate appraisal models considering prior information are better than models based only on sample information. The main aim of the article is to compare models applying prior knowledge (mixed, Bayesian and IRLS) with models referring only to sample data (OLS, ridge and LASSO regression).

\section{Literature review}

There are many quantitative method applications in real estate mass appraisal (Kauko \& d'Amato, 2008; Jahanshiri et al., 2011; d'Amato, 2017; Pagourtzi et al., 2003; McCluskey et al., 2013; Wang \& Li, 2019).

In (Kauko \& d'Amato, 2008), appraisal methods were divided into four groups: model-driven methods, data-driven methods, methods based on machine learning and expert methods. Model-driven methods contain econometric, hedonic and spatial econometric models. Econometric models in appraisals were also considered 
(Benjamin et al., 2004; Isakson, 1998; Dell, 2017; Doszyń, 2020a). Parametric, semiparametric, and nonparametric estimations were considered in (Pace, 1995).

Econometric models with spatial effects were discussed in (Bourassa et al., 2009; Fik et al., 2003). Spatial effects are often treated as a proxy for location. Data-driven methods include nonparametric models (e.g., geographically weighted regression). Machine learning methods were analyzed in (Zurada et al., 2011). Furthermore, an example of an expert system might be found in (Kilpatrick, 2011).

In (Jahanshiri et al., 2011), mass valuation methods were divided into nonspatial and spatial models. Spatial methods were categorized into SAR (spatial autoregressive) models, local models (e.g., geographically weighted regression) and geostatistical models (e.g., kriging). AVMs (automated valuation models) were discussed in (d'Amato, 2017) and (Sing et al., 2021).

In (Pagourtzi et al., 2003), mass appraisal methods were classified into traditional methods (multiple regression, comparable, cost, income, profit, contractors' methods) and advanced methods (artificial neural networks, hedonic models, spatial analysis, fuzzy logic, and ARIMA models). In McCluskey et al. (2013), multiple regression, a spatial lag model, geographically weighted regression and artificial neural networks were compared. Additionally, many useful prediction accuracy measures were suggested.

The possibility of applying prior information in estimation processes was considered many years ago (Durbin, 1953). A systematic formulation of mixed estimation was proposed in (Theil \& Goldberger, 1961; Theil, 1963). The finite sample properties of mixed estimators were discussed in (Swamy \& Mehta, 1969; Mehta \& Swamy, 1970). In (Nagar \& Kakwani, 1964), the bias and moment matrix of mixed estimators were analyzed. The application of mixed estimation in real estate valuation was discussed in (Doszyń, 2021). In this article, a mixed estimator provided not only more efficient estimates but also better real estate value predictions.

In (Mittelhammer \& Conway, 1988), the validity of the Theil-Goldberger mixed estimator was questioned because of the lack of strict rules for applying prior knowledge. It was stated that there was no theoretical basis for a prior specification. Thus, a prior integrated mixed estimator (PIME) was suggested. This is a mixed estimator integrated with respect to the prior distribution. Additionally, the rules of applying prior beliefs were discussed.

The methodological aspects of the Bayesian estimation of econometric models were presented in Koop (2003), together with some examples from the real estate market. In mass appraisals, models other than Bayesian regression are usually used, e.g., in (Del Giudice et al., 2017), the Bayesian approach based on numerical integration on the weight space with the Markov chain hybrid Monte Carlo method (MCHMCM) is recommended.

Inequality restricted least squares (IRLS) models were discussed in (Lovell \& Prescott, 1970; Judge \& Takayama, 1966; Liew, 1976; Zellner, 1961). More advanced tests and considerations regarding IRLS were presented in (Wolak, 1989; Grömping, 2010). The IRLS models of real estate mass appraisal are discussed in (Pace \& Gilley, 1990). In this article, IRLS was compared with OLS by means of Monte Carlo experiments. It was shown that applying inequality restrictions increases estimation efficiency. IRLS also reduces the negative effects of 
multicollinearity in mass appraisal models. In (Doszyń, 2020), IRLS was applied in the mass valuation of undeveloped land properties. It was shown that the IRLS model outperforms OLS results in most domains. In (Liew, 1976), it was stated that IRLS models ensure the consistency of the theory and empirical results. Additionally, the superiority of IRLS over OLS models in terms of efficiency was indicated. More advanced problems regarding IRLS were described in (Werner, 1990; Toker et al., 2013).

The methodological aspects of ridge regression and LASSO were described in (James et al., 2021). Ridge regression in real estate appraisal models was considered in (Newell, 1982; Anderson, 1981; Moore et al., 1984). The application of multiple regression and machine learning methods in real estate mass appraisal was presented in (Gnat \& Doszyń, 2020). In this article, the regularization of ridge regression was also applied.

\section{Methodology}

In the article, six econometric models were considered: OLS, IRLS, mixed, Bayesian, ridge regression and LASSO. All of the models have the same dependent variable (real estate value) and explanatory variables (real estate features). In three models (IRLS, mixed and Bayesian), expert prior knowledge is used. The models are verified with regard to formal criteria and prediction accuracy.

The log-linear econometric model is the starting point

$$
\ln \left(y_{i}\right)=\alpha_{0}+\sum_{k=1}^{K} \sum_{p=2}^{k_{p}} \alpha_{k p} x_{k p i}+\sum_{j=2}^{J} \beta_{j} l_{j i}+u_{i}
$$

where

$y_{i} \quad$ unit market value of $i$-th real estate,

$N$ number of real estate $(i=1,2, \ldots, N)$,

$J$ number of location attractiveness zones $(j=2,3, \ldots, J)$,

$\alpha_{0}$ intercept,

$K \quad$ number of real estate features,

$k_{p} \quad$ number of states of the $k$-th feature,

$\alpha_{k p} \quad$ impact of $p$-th state of feature $k$,

$x_{k p i} \quad$ zero-one variable for $p$-th state of feature $k$,

$\beta_{j} \quad$ location impact for $j$-th zone,

$l_{j i} \quad$ dummy variable for $j$-th location attractiveness zone,

$u_{i} \quad$ error term.

The natural logarithm of real estate unit value is a dependent variable. Real estate values are defined by certified appraisers in individual valuations. A question might be asked why real estate values, but not transaction prices, are a dependent variable. There are a few reasons. First, the number of transactions in a given area may be very small, sometimes even zero. Prices are then not available, while real estate 
values may be determined by appraisers, for example, on the basis of information in adjacent areas. Second, transactions often refer to very similar real estate, which leads to a lack of feature variability. In individual valuations, different properties might be chosen, resulting in increasing variability.

Real estate features are qualitative variables that are measured on an ordinal scale. They were implemented into the models as dummy variables for each feature state. The worst feature states and worst location attractiveness zone were omitted because there was an intercept in the model. Furthermore, the removed variables constitute a point of reference.

Location attractiveness zones were defined by experts as areas with similar location impacts. Therefore, the location effect is estimated by means of dummy variables for each zone, but the worst effect is omitted (because of the intercept). The skipped location zone constitutes a point of reference.

The foregoing model might be presented in matrix form:

$$
\mathbf{y}=\mathbf{X} \boldsymbol{\beta}+\mathbf{u}
$$

where

$\mathbf{y}$ vector of logarithmized unitary real estate values,

X matrix of 0-1 variables for the feature states and location attractiveness zones,

$\boldsymbol{\beta}$ parameter vector of feature states and location impact,

u error term.

In the matrix notation, all parameters are in vector $\boldsymbol{\beta}$, both for feature states and location. $\mathbf{X}$ includes a column with ones (intercept) and dummy variables for feature states and location attractiveness zones.

It should be pointed out that databases from local, often small and inefficient real estate markets are in most cases unsatisfactory from the econometric point of view. Additionally, the variability of explanatory variables is usually low. If all real estate has the same feature states, there is no variability. Moreover, some explanatory variables are qualitative; hence, they are, by definition, subjectively measured. All of these factors make sample information insufficient to properly estimate most econometric models.

In this article, it is stated that sample information might be supported by prior knowledge provided by experts. If the sample information is not adequate, it can be enriched by prior knowledge. In the presented research, prior knowledge will be used in the mixed, Bayesian and IRLS models.

Sample information is described by a linear regression model (2). In the mixed model, prior information has the form of stochastic restrictions imposed on the parameters:

$$
\mathbf{r}=\mathbf{R} \boldsymbol{\beta}+\mathbf{v}
$$

where

$\mathbf{R}$ restriction matrix $[j \times P]$, 
$j \quad$ number of restrictions,

$P \quad$ number of parameters,

$\mathbf{r}$ prior expected parameter values $[j \times 1]$,

$\mathbf{v}$ prior values of parameter errors $[j \times 1]$.

Matrix $\mathbf{R}$ includes restrictions. In the described model, restrictions relate to parameters next to dummy variables for real estate feature states. In the mixed model, restrictions are not imposed on intercept and parameters next to dummies for location. There are $j$ restrictions; hence, matrix $\mathbf{R}$ has $j$ rows. $\mathbf{R}$ has $P$ columns, where $P$ is the number of model parameters. Columns for parameters without restrictions (intercept and parameters next to dummies for location) contain only zeros. Prior information included in $\mathbf{r}$ and $\mathbf{v}$ may come from experts, different samples or personal beliefs. It is assumed that error vectors $\mathbf{v}$ and $\mathbf{u}$ are independent and have a known covariance matrix $E(\mathbf{v v})^{\prime}=\Psi$.

A combination of sample (2) and prior information (3) provides:

$$
\left[\begin{array}{l}
y \\
\mathbf{r}
\end{array}\right]=\left[\begin{array}{l}
\mathbf{X} \\
\mathbf{R}
\end{array}\right][\boldsymbol{\beta}]+\left[\begin{array}{l}
\mathbf{u} \\
\mathbf{v}
\end{array}\right]
$$

As mentioned before, in a mixed model, restrictions are imposed only on parameters for real estate feature states (without intercept and parameters next to dummies for location).

An error covariance matrix might be expressed as:

$$
E\left[\left[\begin{array}{l}
\mathbf{u} \\
\mathbf{v}
\end{array}\right]\left[\begin{array}{ll}
\mathbf{u}^{\prime} & \mathbf{v}^{\prime}
\end{array}\right]\right]=\left[\begin{array}{cc}
\sigma^{2} \mathbf{I} & \mathbf{0} \\
\mathbf{0} & \boldsymbol{\Psi}
\end{array}\right]
$$

where $\sigma^{2}$ is the variance in the error term. Off-diagonal blocks are zero matrices; hence, sample and prior error vectors are independent. Additionally, the homoskedasticity of $\sigma^{2}$ is assumed. A dependent variable is in logs, which often reduces heteroskedasticity (if present).

A generalized least squares mixed estimator might be expressed as

$$
\begin{gathered}
\mathbf{b}_{m}=\left[\left[\begin{array}{l}
\mathbf{X} \\
\mathbf{R}
\end{array}\right]^{\prime}\left[\begin{array}{cc}
\sigma^{2} \mathbf{I} & \mathbf{0} \\
\mathbf{0} & \boldsymbol{\Psi}
\end{array}\right]^{-1}\left[\begin{array}{l}
\mathbf{X} \\
\mathbf{R}
\end{array}\right]\right]^{-1}\left[\begin{array}{l}
\mathbf{X} \\
\mathbf{R}
\end{array}\right]^{\prime}\left[\begin{array}{cc}
\sigma^{2} \mathbf{I} & \mathbf{0} \\
\mathbf{0} & \boldsymbol{\Psi}
\end{array}\right]^{-1}\left[\begin{array}{l}
\mathbf{y} \\
\mathbf{r}
\end{array}\right] \\
\mathbf{b}_{m}=\left[\sigma^{-2} \mathbf{X}^{\prime} \mathbf{X}+\mathbf{R}^{\prime} \Psi^{-1} \mathbf{R}\right]^{-1}\left[\sigma^{-2} \mathbf{X}^{\prime} \mathbf{y}+\mathbf{R}^{\prime} \Psi^{-1} \mathbf{r}\right]
\end{gathered}
$$

and the matrix of $\mathbf{b}_{m}$ covariances is

$$
\mathbf{V}\left(\mathbf{b}_{m}\right)=\left[\sigma^{-2} \mathbf{X}^{\prime} \mathbf{X}+\mathbf{R}^{\prime} \Psi^{-1} \mathbf{R}\right]^{-1}
$$

Error homoskedasticity was assumed: $E\left(\mathbf{u u}^{\prime}\right)=\sigma^{2} \mathbf{I}$, but a more general form of the error covariance matrix $E\left(\mathbf{u u}^{\prime}\right)=\boldsymbol{\Omega}$ might also be applied.

Because $\sigma^{2}$ is not known, an ordinary least squares estimator is applied, which results in an estimate of the residual variance 


$$
s^{2}=\left[\mathbf{y}^{\prime} \mathbf{y}-\mathbf{y}^{\prime} \mathbf{X}\left[\mathbf{X}^{\prime} \mathbf{X}\right]^{-1} \mathbf{X}^{\prime} \mathbf{y}\right] /(N-P)
$$

In mixed estimation, it is often interesting whether sample and prior information are consistent or what are the shares of each component (sample and prior) in posterior precision. Many suggestive proposals of that type are presented in (Theil, 1963).

The comparability statistic of the sample and prior information has an asymptotic $\chi^{2}$ distribution with $P$ degrees of freedom and might be obtained as:

$$
\hat{\gamma}=[\mathbf{r}-\mathbf{R b}]^{\prime}\left[s^{2} \mathbf{R}[\mathbf{X} / \mathbf{X}]^{-\mathbf{1}} \mathbf{R}^{\prime}+\mathbf{\Psi}\right]^{-1}[\mathbf{r}-\mathbf{R b}]
$$

where $\mathbf{b}=\left[\mathbf{X}^{\prime} \mathbf{X}\right]^{-1} \mathbf{X}^{\prime} \mathbf{y}$ is the OLS estimator using sample information.

Shares of prior $\left(\theta_{p}\right)$ and sample $\left(\theta_{s}\right)$ information can be expressed as follows (Theil, 1963):

$$
\begin{aligned}
& \theta_{p}=\frac{1}{P} \operatorname{tr} \mathbf{R}^{\prime} \Psi^{-1} \mathbf{R}\left[s^{-2} \mathbf{X} / \mathbf{X}+\mathbf{R}^{\prime} \Psi^{-1} \mathbf{R}\right]^{-1} \\
& \theta_{s}=\frac{1}{P} t r s^{-2} \mathbf{X}^{\prime} \mathbf{X}\left[s^{-2} \mathbf{X} / \mathbf{X}+\mathbf{R}^{\prime} \Psi^{-1} \mathbf{R}\right]^{-1}
\end{aligned}
$$

In (11) and (12) "tr" stands for a matrix trace.

The Bayesian model also gives the possibility to apply prior knowledge. In the Bayesian model, the natural conjugate normal-gamma prior was used (Koop, 2003):

$$
\boldsymbol{\beta}, h \sim N G\left(\begin{array}{l}
\boldsymbol{\beta}, \mathbf{V}, s^{-2}, v \\
--_{-}
\end{array}\right)
$$

where

及 prior expected parameter values vector,

$\bar{s}^{2} \quad$ prior disturbance variance,

$\bar{s}^{-2} \mathbf{V}$ inverse of prior covariance matrix of parameters,

$\bar{v}-$ prior parameter related to degrees of freedom.

Vector $\boldsymbol{\beta}$ conditional on $h=s^{-2}$ is a multivariate normal and can be written as

$$
\beta \mid h \sim N\left(\boldsymbol{\beta}, h^{-1} \mathbf{V}\right)
$$

Error precision $h$ has the gamma prior and can be presented as

$$
h \sim G\left(s_{-}^{-2}, v\right)
$$

After taking into account (14) and (15), we have the natural conjugate normalgamma prior of form (13). Hyperparameters $\boldsymbol{\beta}, \mathbf{V}, s^{-2}, v$ are chosen by the researcher. 
They represent prior knowledge. Vector $\boldsymbol{\beta}$ contains prior means for regression coefficients, while $\mathbf{V}$ is a positive definite prior covariance matrix.

The posterior is a product of the likelihood function and the prior after collecting terms. Assuming multivariate normal density, the likelihood function has the following form:

$$
p(\mathbf{y} \mid \boldsymbol{\beta}, h)=\frac{1}{(2 \pi)^{\frac{N}{2}}}\left\{h^{\frac{1}{2}} \exp \left[-\frac{h}{2}[\boldsymbol{\beta}-\hat{\boldsymbol{\beta}}] \mathbf{X}^{\prime} \mathbf{X}[\boldsymbol{\beta}-\hat{\boldsymbol{\beta}}]\right]\right\}\left\{h^{\frac{v}{2}} \exp \left[-\frac{h v}{2 s^{-2}}\right]\right\}
$$

where

$$
\begin{gathered}
v=N-P \\
\hat{\boldsymbol{\beta}}=\left[\mathbf{X}^{\prime} \mathbf{X}\right]^{-1} \mathbf{X}^{\prime} \mathbf{y} \\
s^{2}=\frac{[\mathbf{y}-\mathbf{X} \hat{\boldsymbol{\beta}}]^{\prime}[\mathbf{y}-\mathbf{X} \hat{\boldsymbol{\beta}}]}{v}
\end{gathered}
$$

The above estimates $\left(\hat{\boldsymbol{\beta}}, s^{2}, v\right)$ are the same as in OLS. The form of (16) indicates that the natural conjugate prior is normalgamma. Combining prior and likelihood gives a posterior normal-gamma distribution of the following form (Koop, 2003):

$$
\boldsymbol{\beta}, h \mid y \sim N G\left(\overline{\boldsymbol{\beta}}, \overline{\mathbf{V}}, \bar{s}^{-2}, \bar{v}\right)
$$

where

$$
\begin{gathered}
\overline{\mathbf{V}}=\left(\begin{array}{c}
\mathbf{V}^{-1}+\mathbf{X}^{\prime} \mathbf{X} \\
-
\end{array}\right)^{-1} \\
\overline{\boldsymbol{\beta}}=\overline{\mathbf{V}}\left(\begin{array}{c}
\mathbf{V}^{-1} \boldsymbol{\beta}+\mathbf{X}^{\prime} \mathbf{X} \hat{\boldsymbol{\beta}} \\
-
\end{array}\right) \\
\bar{v}=v+N
\end{gathered}
$$

The above expression (17) describes the joint posterior distribution. It is a combination of prior and sample information. Posterior error precision $\bar{s}^{-2}$ is estimated on the basis of

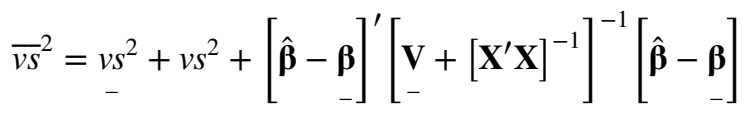

The above expressions present a joint posterior distribution. After integration, the marginal posterior for $\boldsymbol{\beta}$ might be obtained. It is a multivariate t distribution 


$$
\boldsymbol{\beta} \mid y \sim t\left(\overline{\boldsymbol{\beta}}, \bar{s}^{2} \overline{\mathbf{V}}, \bar{v}\right)
$$

In the Bayesian model estimation, analytical results following the definition of the $\mathrm{t}$ distribution are used:

$$
\begin{gathered}
E(\boldsymbol{\beta} \mid \mathbf{y})=\overline{\boldsymbol{\beta}} \\
\operatorname{var}(\boldsymbol{\beta} \mid \mathbf{y})=\frac{\overline{v s}^{2}}{v-2} \overline{\mathbf{V}}
\end{gathered}
$$

Expressions (23) and (24) are applied in the presented research.

In the IRLS model, restrictions in the form of inequalities are imposed on the parameters. IRLS is estimated by minimizing the sum of the squared errors:

$$
\mathbf{u}^{\mathbf{T}} \mathbf{u}=[\mathbf{y}-\mathbf{X} \boldsymbol{\beta}]^{\mathbf{T}}[\mathbf{y}-\mathbf{X} \boldsymbol{\beta}] \rightarrow \min
$$

with imposed restrictions

$$
\mathbf{Z} \boldsymbol{\beta}^{\mathbf{r}} \geq \mathbf{0}
$$

where

$\boldsymbol{\beta}^{\mathbf{r}}$ subvector with parameters next to dummies for feature state impact,

$\mathbf{Z}$ matrix of restrictions imposed on subvector $\boldsymbol{\beta}^{\mathbf{r}}$.

Matrix $\mathbf{Z}$ has as many rows as restrictions. The number of columns is equal to the number of parameters $\alpha_{k p}$ in (1). Relations (25) and (26) constitute quadratic programming tasks.

In the IRLS model, a nonnegative impact of the feature states $\left(\alpha_{k p} \geq 0\right)$ and monotonicity of the feature impact $\left(\alpha_{k, p+1} \geq \alpha_{k p}\right)$ were assumed. Feature states should increase unitary real estate value, hence, restriction imposing nonnegativity. Monotonicity means that a better feature state $(p+1)$ should increase a unitary real estate value not less than the preceding state $(p)$. Restrictions are not imposed on intercept and parameters next to dummies for location because, according to expert knowledge, the location impact should be highly positive.

In ridge regression and LASSO, the parameters generally shrink toward zero. This might decrease the parameter variance; hence, the estimator's efficiency could be higher. Ridge regression and LASSO are very similar to OLS, but a slightly different quantity is minimized.

Referring to the OLS model (1), in ridge regression, the following sum of squares is minimized:

$$
\sum_{i=1}^{N}\left(\ln \left(y_{i}\right)-\alpha_{0}-\sum_{k=1}^{K} \sum_{p=2}^{k_{p}} \alpha_{k p} x_{k p i}-\sum_{j=2}^{J} \beta_{j} l_{j i}\right)^{2}+\lambda\left(\sum_{k=1}^{K} \sum_{p=2}^{k_{p}} \alpha_{k p}^{2}+\sum_{j=2}^{J} \beta_{j}^{2}\right)
$$

where $\lambda \geq 0$. 
The first part is the same as in the OLS model, while the second part is a penalty that decreases all of the parameters (except intercept) toward zero. If $\lambda=0$, ridge regression and OLS coefficients are the same. If $\lambda$ is high, the coefficients are close to zero.

LASSO is very similar to ridge regression, but as a penalty, absolute values of the parameters are used. The following sum of squares is minimized:

$$
\sum_{i=1}^{N}\left(\ln \left(y_{i}\right)-\alpha_{0}-\sum_{k=1}^{K} \sum_{p=2}^{k_{p}} \alpha_{k p} x_{k p i}-\sum_{j=2}^{J} \beta_{j} l_{j i}\right)^{2}+\lambda\left(\sum_{k=1}^{K} \sum_{p=2}^{k_{p}}\left|\alpha_{k p}\right|+\sum_{j=2}^{J}\left|\beta_{j}\right|\right)
$$

In the LASSO, some coefficients might be equal to zero; therefore, variable selection is performed.

\section{Data and empirical results}

In the present study, undeveloped land properties for residential purposes in Szczecin (Poland) are analyzed. Generally, the research consists of two main stages. First, information on 117 comparable real properties was used to train the econometric models (training dataset). The comparable real properties were randomly chosen from all real properties subjected to mass appraisal in the considered area. Their market values were known because they were evaluated in individual appraisals by property appraisers. The number of comparables should not be too high because values are obtained in individual valuations, which might be costly. All of the data referred to the year 2018 .

In the second stage, the estimated models were applied in the valuation of 318 real estate properties from the test dataset. The market values of these properties were also known because they were determined in individual valuations by property experts.

The valuation area was divided into sixteen location attractiveness zones. The training set included 117 randomly drawn real estate from 16 zones. The test set contained 318 undeveloped land properties from 3 zones.

In Table 1, real estate features and their states are presented.

Almost all of the features are qualitative variables, measured on an ordinal scale. They were implemented into econometric models as dummy variables for each feature state. The worst variants were omitted due to strict collinearity (there are intercepts in the models). Moreover, only one feature (neighborhood) has four states. The remaining features have three categories, which can be generally described as worst, average and favorable.

Land area is a quantitative variable; however, it was also measured on an ordinal scale with three states: large, average, and small. In addition, scale weakening was suggested by real estate analysts because land area is frequently perceived in this manner by market participants.

The unitary value of undeveloped land is a dependent variable. Models in exponential forms are estimated; therefore, an explained variable is in natural logs. Unitary real estate values in the training set were in the range of $174,80-680,73$ PLNs 
Table 1. Real estate features

\begin{tabular}{ll}
\hline Feature & Feature state/symbol \\
\hline Land area & $\begin{array}{l}\text { large } \\
\text { average }-a_{1} \\
\text { small }-a_{2}\end{array}$ \\
none & incomplete $-u_{1}$ \\
Utilities & complete $-u_{2}$ \\
& unfavorable \\
Transport availability & average $-t a_{1}$ \\
& favorable $-t a_{2}$ \\
Neighborhood & onerous \\
& unfavorable $-n_{1}$ \\
average $-n_{2}$ \\
favorable $-n_{3}$ \\
Physical properties & unfavorable \\
& average $-p p_{1}$ \\
favorable $-p p_{2}$
\end{tabular}

(Polish zlotys) with a median value equal to 502,11 PLNs. The first and third quartiles were equal to 302,04 PLNs and 586,63 PLNs, respectively. The property feature distributions of 117 comparable real properties are presented below in Table 2. Real estate features were coded such that the worst variant was 0 , the next variant was 1 , etc.

The first conclusion is that favorable states (appointed as 2) dominated. For example, $91.5 \%$ of the real estate has complete utilities. In the case of remaining features, favorable states (marked as 2) were present in more than $50 \%$ of the real estate. The worst feature states were in the minority.

These real properties constitute a training set; they were from sixteen location attractiveness zones. Zones were introduced into the econometric models as dummy variables. Furthermore, a dummy for the third location attractiveness zone was omitted to avoid strict collinearity. According to expert knowledge, in this zone, unitary property values were the lowest.

For the training set, six econometric models were estimated: ordinary least squares (OLS), mixed model (ME), Bayesian model (BE), inequality restricted least

Table 2. Feature distributions (shares of feature states) for a training set (117 comparable real estate)

\begin{tabular}{llllll}
\hline Feature state & Surface area & Utilities & $\begin{array}{l}\text { Transport avail- } \\
\text { ability }\end{array}$ & Neighborhood & $\begin{array}{l}\text { Physical } \\
\text { proper- } \\
\text { ties }\end{array}$ \\
\hline 0 & 0.179 & 0.026 & 0.060 & 0.051 & 0.103 \\
1 & 0.274 & 0.060 & 0.376 & 0.085 & 0.393 \\
2 & 0.547 & 0.915 & 0.564 & 0.607 & 0.504 \\
3 & $-{ }^{\mathrm{a}}$ & - & - & 0.256 & - \\
\hline
\end{tabular}

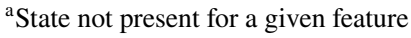


squares (IRLS) model, ridge regression (RR) and LASSO. In mixed and Bayesian estimation, specific prior knowledge was used. It was given by real estate appraisers, who provided parameter ranges for real estate feature state impact.

Real estate appraisers from local markets have to use the weights of real estate features in individual valuations (in the comparative approach). Therefore, several appraisers were asked about those kinds of weights. Appraisers often valued real estate in individual valuations; hence, they knew the weights for real estate features well. These weights were transformed into parameter intervals for each real estate feature state. The team of appraisers consisted of several people. Each appraiser had experience in the local market. A consensus was made (by the appraisers) according to the prior intervals for the model parameters. The cost of collecting prior information from the appraisers was not high. They previously evaluated considerable number of real estate from local markets. After this, the appraisers were asked to give prior information in the form of parameter intervals. This kind of information was also provided by appraisers from the local market, who often knew the weights of real estate features from practice and, thus, could give their parameter intervals.

Next, the multiplicative impact of weights was assumed. For example, if the appraisers stated that the impact of land area on real estate value is between 0 and $15 \%$, then it was assumed that the prior parameter intervals for these two states (average, small) are $0-0.075 \%$ (average area) and $0.075 \%-0.15 \%$ (small area). Analogous intervals were provided by the appraisers for other features and their states. This kind of prior knowledge was used in the estimation of the mixed and Bayesian models.

In mixed estimation, parameter intervals were defined for all parameters without the constant term and parameters for location attractiveness zones. Experts did not have prior knowledge due to the constant term and location attractiveness zones. In the Bayesian estimation, prior distributions have to be defined for all model parameters, which is also the case for intercept and parameters next to dummies for location zones. Therefore, experts gave parameter intervals needed in the Bayesian model but with the condition that these priors are rather opaque. Priors were generally provided for the impact of real estate feature states. For the Bayesian model, additional prior information was required, and it was given by experts but with much less conviction.

Generally, in mixed and Bayesian estimation, the same prior information was used to make the results comparable. The difference was that in the Bayesian model, priors were also provided for intercept and location, which was not necessary in mixed estimation.

Real estate appraisers gave their parameter intervals $\langle a, b\rangle$. It was assumed that the provided intervals cover parameters with a probability equal to 0.95 . Therefore, the prior expected value was equal to $(a+b) / 2$. The standard deviation equals $(b-a) / 4$; hence, interval ends are located two standard deviations from the expected value.

The prior values used in mixed estimation are presented below in Table 3. The expected values constitute an $\mathbf{r}$ vector in (6). Variances are on the main diagonal of the $\boldsymbol{\Psi}$ matrix in (6).

In Bayesian estimation, the natural conjugate normal-gamma priors were assumed. Model parameters conditional on error precision (inverse variance) are 
Table 3. Prior knowledge used in mixed estimation

\begin{tabular}{|c|c|c|c|c|c|}
\hline \multirow[b]{2}{*}{$a_{1}$} & \multicolumn{2}{|c|}{$\begin{array}{l}\text { Parameter } \\
\text { range }\end{array}$} & \multirow{2}{*}{$\begin{array}{l}\text { Expected value } \\
0.038\end{array}$} & \multirow{2}{*}{$\begin{array}{l}\text { Standard deviation } \\
0.01875\end{array}$} & \multirow{2}{*}{$\begin{array}{l}\text { Variance } \\
0.00035\end{array}$} \\
\hline & 0.000 & 0.075 & & & \\
\hline$a_{2}$ & 0.075 & 0.150 & 0.113 & 0.01875 & 0.00035 \\
\hline$u_{1}$ & 0000 & 0.050 & 0.025 & 0.01250 & 0.00016 \\
\hline$u_{2}$ & 0.050 & 0.100 & 0.075 & 0.01250 & 0.00016 \\
\hline$t a_{1}$ & 0.000 & 0.075 & 0.038 & 0.01875 & 0.00035 \\
\hline$t a_{2}$ & 0.075 & 0.150 & 0.113 & 0.01875 & 0.00035 \\
\hline$n_{1}$ & 0.000 & 0.067 & 0.033 & 0.01667 & 0.00028 \\
\hline$n_{2}$ & 0.067 & 0.133 & 0.100 & 0.01667 & 0.00028 \\
\hline$n_{3}$ & 0.133 & 0.200 & 0.167 & 0.01667 & 0.00028 \\
\hline$p p_{1}$ & 0.000 & 0.050 & 0.025 & 0.01250 & 0.00016 \\
\hline$p p_{2}$ & 0.050 & 0.100 & 0.075 & 0.01250 & 0.00016 \\
\hline
\end{tabular}

then multivariate normal distributions. Moreover, the prior distribution for error precision is gamma.

Prior distributions for real estate feature states are the same as in mixed estimation. In the Bayesian approach, priors must also be provided for intercept and parameters next to dummies for location attractiveness zones. Two parameter intervals for location attractiveness zones were defined by real estate experts. For zones nos. $1-5$, parameters in the range $0-0.2$ were suggested. For the remaining zones, parameter intervals between 0.2 and 0.4 were recommended. These intervals were based on expert knowledge from the local market. This information was not used in mixed estimation because it is more opaque than priors for real estate feature state impacts. The advantage of mixed estimation is that the number of restrictions is not necessarily the same as the number of parameters, meaning that we may use only the information that we have.

Prior information used in the Bayesian approach is presented in Table 4. Expected values constitute the vector $\boldsymbol{\beta}$. Prior error precision is $h=s^{-2}=0.05^{-2}=400$. The variances in Table 4 were divided by $s^{2}$, which gave the main diagonal of the matrix V. Prior value $v=40$, which is approximately $1 / 3$ of the observations $(N=117)$.

Estimation results are presented below in Table 5. In the OLS model, most of the parameters were insignificant $(\alpha=0.05)$. Only neighborhood and location had a significant impact. On the basis of the White test, the hypothesis stating error homoskedasticity could not be rejected $(\alpha=0.05)$.

In mixed estimation, the share of prior and sample information was calculated on the basis of (11)-(12). The share of sample information dominated and was equal to 0.643 . The share of prior information was equal to 0.357 . With regard to (10), it might be stated that the hypothesis regarding the consistency of prior and sample information could not be rejected ( $\mathrm{p}$ value equal to 0.693 ).

The Bayesian model is based on the natural conjugate normal-gamma prior. In that case, an analytical solution is known; therefore, specific packages are not 
Table 4. Prior knowledge used in Bayesian estimation

\begin{tabular}{|c|c|c|c|c|c|}
\hline \multirow{2}{*}{$\begin{array}{l}\text { Variables } \\
\text { const }\end{array}$} & \multicolumn{2}{|c|}{ Parameter range } & \multirow{2}{*}{$\begin{array}{l}\text { Expected value } \\
5.500\end{array}$} & \multirow{2}{*}{$\begin{array}{l}\text { Standard deviation } \\
0.25000\end{array}$} & \multirow{2}{*}{$\begin{array}{l}\text { Variance } \\
0.06250\end{array}$} \\
\hline & 5.000 & 6.000 & & & \\
\hline$a_{1}$ & 0.000 & 0.075 & 0.038 & 0.01875 & 0.00035 \\
\hline$a_{2}$ & 0.075 & 0.150 & 0.113 & 0.01875 & 0.00035 \\
\hline$u_{1}$ & 0.000 & 0.050 & 0.025 & 0.01250 & 0.00016 \\
\hline$u_{2}$ & 0.050 & 0.100 & 0.075 & 0.01250 & 0.00016 \\
\hline$t a_{1}$ & 0.000 & 0.075 & 0.038 & 0.01875 & 0.00035 \\
\hline$t a_{2}$ & 0.075 & 0.150 & 0.113 & 0.01875 & 0.00035 \\
\hline$n_{1}$ & 0.000 & 0.067 & 0.033 & 0.01667 & 0.00028 \\
\hline$n_{2}$ & 0.067 & 0.133 & 0.100 & 0.01667 & 0.00028 \\
\hline$n_{3}$ & 0.133 & 0.200 & 0.167 & 0.01667 & 0.00028 \\
\hline$p p_{1}$ & 0.000 & 0.050 & 0.025 & 0.01250 & 0.00016 \\
\hline$p p_{2}$ & 0.050 & 0.100 & 0.075 & 0.01250 & 0.00016 \\
\hline $\mathrm{sal}_{1}$ & 0.000 & 0200 & 0.100 & 0.05000 & 0.00250 \\
\hline $\mathrm{sal}_{2}$ & 0.000 & 0.200 & 0.100 & 0.05000 & 0.00250 \\
\hline $\mathrm{Sal}_{4}$ & 0.000 & 0.200 & 0.100 & 0.05000 & 0.00250 \\
\hline $\mathrm{sal}_{5}$ & 0.000 & 0.200 & 0.100 & 0.05000 & 0.00250 \\
\hline $\mathrm{sal}_{6}$ & 0.200 & 0.400 & 0.300 & 0.05000 & 0.00250 \\
\hline $\mathrm{sal}_{7}$ & 0.200 & 0.400 & 0.300 & 0.05000 & 0.00250 \\
\hline $\mathrm{sal}_{8}$ & 0.200 & 0.400 & 0.300 & 0.05000 & 0.00250 \\
\hline $\mathrm{sal}_{9}$ & 0.200 & 0.400 & 0.300 & 0.05000 & 0.00250 \\
\hline $\operatorname{sal}_{10}$ & 0.200 & 0.400 & 0.300 & 0.05000 & 0.00250 \\
\hline$s a l_{11}$ & 0.200 & 0.400 & 0.300 & 0.05000 & 0.00250 \\
\hline $\operatorname{sal}_{12}$ & 0.200 & 0.400 & 0.300 & 0.05000 & 0.00250 \\
\hline $\operatorname{sal}_{13}$ & 0.200 & 0.400 & 0.300 & 0.05000 & 0.00250 \\
\hline $\mathrm{sal}_{14}$ & 0.200 & 0.400 & 0.300 & 0.05000 & 0.00250 \\
\hline $\operatorname{sal}_{15}$ & 0.200 & 0.400 & 0.300 & 0.05000 & 0.00250 \\
\hline $\operatorname{sal}_{16}$ & 0.200 & 0.400 & 0.300 & 0.05000 & 0.00250 \\
\hline
\end{tabular}

necessary. The IRLS model was estimated with the use of the ic.infer package available in the R. Ridge regression and LASSO models was estimated in the glmnet R package. All explanatory variables were dummies; hence, they were not standardized (all of the coefficients are comparable). In ridge regression and LASSO, proper $\lambda$ values should be chosen. It was computed on the basis of 10 -fold cross-validation for the training set (117 comparable real estate properties) and was equal to 0.011 (RR) and 0.012 (LASSO).

The estimation results for all of the considered models are presented below in Table 5.

Estimates in mass valuation models should be consistent with theoretical expectations. In the presented models, variables are defined such that parameter estimates have to be positive. Additionally, the impact of a better feature state 
Table 5. Estimation results

\begin{tabular}{|c|c|c|c|c|c|c|}
\hline & OLS & $\mathrm{ME}$ & $\mathrm{BE}$ & IRLS & RR & LASSO \\
\hline const & $5.463^{b}$ & 5.227 & 5.417 & 5.098 & 5.979 & 5.955 \\
\hline$a_{1}$ & $-0.038^{c}$ & 0.037 & -0.001 & 0.014 & -0.121 & -0.112 \\
\hline$a_{2}$ & -0.005 & 0.107 & 0.050 & 0.031 & -0.038 & 0.000 \\
\hline$u_{1}$ & -0.034 & 0.030 & 0.062 & 0.015 & 0.019 & 0.000 \\
\hline$u_{2}$ & -0.239 & 0.067 & 0.005 & 0.031 & -0.081 & 0.000 \\
\hline$t a_{1}$ & 0.141 & 0.038 & 0.071 & 0.167 & 0.061 & 0.000 \\
\hline$t a_{2}$ & 0.176 & 0.117 & 0.150 & 0.248 & 0.132 & 0.099 \\
\hline$n_{1}$ & -0.042 & 0.027 & -0.012 & 0.017 & -0.101 & -0.072 \\
\hline$n_{2}$ & 0.228 & 0.110 & 0.149 & 0.258 & 0.075 & 0.000 \\
\hline$n_{3}$ & 0.251 & 0.165 & 0.167 & 0.262 & 0.073 & 0.000 \\
\hline$p p_{1}$ & -0.034 & 0.022 & 0.002 & 0.022 & -0.042 & 0.000 \\
\hline$p p_{2}$ & 0.054 & 0.077 & 0.073 & 0.080 & 0.018 & 0.000 \\
\hline$l_{1}$ & 0.238 & 0.085 & 0.024 & 0.216 & -0.105 & 0.000 \\
\hline$l_{2}$ & 0.130 & 0.154 & 0.022 & 0.106 & -0.095 & 0.000 \\
\hline$l_{4}$ & 0.190 & 0.093 & 0.009 & 0.171 & -0.129 & 0.000 \\
\hline$l_{5}$ & 0.122 & 0.180 & 0.044 & 0.181 & -0.139 & 0.000 \\
\hline$l_{6}$ & 0.226 & 0.227 & 0.123 & 0.264 & -0.089 & 0.000 \\
\hline$l_{7}$ & 0.290 & 0.329 & 0.197 & 0.353 & -0.067 & 0.000 \\
\hline$l_{8}$ & 0.364 & 0.373 & 0.238 & 0.381 & -0.017 & 0.000 \\
\hline$l_{9}$ & 0.783 & 0.741 & 0.588 & 0.768 & 0.260 & 0.232 \\
\hline$l_{10}$ & 0.679 & 0.700 & 0.530 & 0.703 & 0.181 & 0.122 \\
\hline$l_{11}$ & 0.278 & 0.327 & 0.190 & 0.318 & -0.071 & 0.000 \\
\hline$l_{12}$ & 0.643 & 0.626 & 0.459 & 0.642 & 0.111 & 0.000 \\
\hline$l_{13}$ & 0.776 & 0.738 & 0.584 & 0.767 & 0.253 & 0.237 \\
\hline$l_{14}$ & 0.737 & 0.784 & 0.590 & 0.741 & 0.240 & 0.253 \\
\hline$l_{15}$ & 0.792 & 0.751 & 0.588 & 0.767 & 0.250 & 0.218 \\
\hline$l_{16}$ & 0.860 & 0.698 & 0.505 & 0.830 & 0.123 & 0.000 \\
\hline$S e^{2}$ & 0.042 & 0.052 & 0.048 & 0.049 & 0.066 & 0.087 \\
\hline$R^{2}$ & 0.729 & 0.664 & 0.687 & 0.681 & 0.573 & 0.437 \\
\hline
\end{tabular}

${ }^{\mathrm{b}}$ In the OLS model, estimates significant at the 0.05 level are shown in bold

${ }^{\mathrm{c}}$ Nonpositive coefficients are in italics

should be higher than the influence of the preceding state, which might be called the monotonicity of feature impact.

These criteria were not fulfilled by the OLS, ridge regression and LASSO models. In these models, the impact of many variables was negative. Additionally, the influence of a better feature state was often lower than the impact of the previous state. In LASSO most of the estimates were equal to zero. Additionally, in ridge regression and LASSO, the impact of location was frequently negative or equal to zero. These kinds of results were very questionable from a practical point of view. Many zeros in LASSO were probably the result of setting $\lambda$ by cross-validation. In 
cross-validation, it is likely that some estimates are equal to zero. Therefore, LASSO does not work well for those kinds of datasets. There were only 117 observations and as many as 16 location zones. Ridge regression is also not applicable for those kinds of databases.

In the Bayesian model, the impact of $a_{1}$ and $n_{1}$ was negative but very close to zero. Furthermore, monotonicity might not be attributed to utilities because the impact of $u_{2}$ was lower than $u_{1}$.

In the case of mixed estimation and IRLS, the impact of feature states was positive and monotonic; hence, the estimation results were consistent with expectations. For OLS, IRLS, mixed and Bayesian estimation, the influence of location was high and positive, which was also confirmed by real estate experts having experience from the local market.

The OLS, Bayesian, IRLS and mixed models had a determination ratio in the range 0.664-0.729. The determination coefficient was computed for delogarithmized unitary real estate values. In addition, the determination ratios for ridge regression and LASSO were equal, 0.573 and 0.437 , respectively.

In Table 6, below the standard errors for the OLS, mixed and Bayesian models are presented. For IRLS, ridge regression and LASSO, the standard errors are not reported. Generally, standard errors were smaller when priors were used. Errors were the highest for the OLS model. Standard errors were the smallest for mixed estimation but only for parameters for which prior knowledge was applied. For mixed estimation, priors were not used for location, and in those cases, standard errors were smaller for the Bayesian model. As mentioned before, in the Bayesian model, priors were also applied for location, and the standard error for the intercept was the smallest for mixed estimation, although the prior was not used.

Model estimation was the first step; however, the main aim was to verify how good the predictions are. Therefore, all of the estimated models were applied in the evaluation of 318 real estate properties from the test set, and the property values were also known because all of the properties were evaluated in individual appraisals.

In the test set, unitary values were in the range of 502.11-701.43 PLN with the median equal to 592.28 PLN. The first and third quartiles were equal to 569.04 and 623.53 PLN. The characteristics of the features for the real estate for this dataset can be seen below in Table 7. Feature distributions were quite similar to those for the training set. Generally, better feature states were most frequent. All of the real estate had complete utilities, which was the best variant. In contrast, for neighborhoods, the average variant dominated.

Predictive accuracy was judged with respect to percentage errors $\left(P E_{i}=\left(w_{i}-\hat{w}_{i}\right) / w_{i}\right)$ and absolute percentage errors $\left(A P E_{i}=\left|w_{i}-\hat{w}_{i}\right| / w_{i}\right)$, where $w_{i}$ is a unitary real estate value obtained in individual valuations and $\hat{w}_{i}$ are theoretical values from a given model. As many as 318 PE and APE were calculated.

The characteristics of these two errors can be seen below in Tables 8 and 9 .

It is better if minimum, first decile and first quartile of PE are negative and closer to zero. These values inform us about overestimation. The minimum for IRLS gave a theoretical real estate value that was overestimated by $18.3 \%$. For LASSO, the minimum was positive; hence, all theoretical values were lower than empirical values. 
Table 6. Standard errors for the OLS, mixed and Bayesian models

\begin{tabular}{|c|c|c|c|}
\hline & OLS & $\mathrm{ME}$ & $\mathrm{BE}$ \\
\hline const & 0.234 & 0.083 & 0.096 \\
\hline$a_{1}$ & 0.077 & 0.018 & 0.049 \\
\hline$a_{2}$ & 0.063 & 0.017 & 0.043 \\
\hline$u_{1}$ & 0.155 & 0.012 & 0.044 \\
\hline$u_{2}$ & 0.141 & 0.012 & 0.043 \\
\hline$t a_{1}$ & 0.095 & 0.017 & 0.050 \\
\hline$t a_{2}$ & 0.101 & 0.017 & 0.051 \\
\hline$n_{1}$ & 0.117 & 0.011 & 0.040 \\
\hline$n_{2}$ & 0.103 & 0.020 & 0.049 \\
\hline$n_{3}$ & 0.110 & 0.016 & 0.049 \\
\hline$p p_{1}$ & 0.071 & 0.012 & 0.037 \\
\hline$p p_{2}$ & 0.070 & 0.012 & 0.036 \\
\hline$l_{1}$ & 0.164 & 0.143 & 0.111 \\
\hline$l_{2}$ & 0.152 & 0.143 & 0.110 \\
\hline$l_{4}$ & 0.130 & 0.121 & 0.092 \\
\hline$l_{5}$ & 0.120 & 0.105 & 0.081 \\
\hline$l_{6}$ & 0.121 & 0.114 & 0.087 \\
\hline$l_{7}$ & 0.114 & 0.099 & 0.074 \\
\hline$l_{8}$ & 0.113 & 0.110 & 0.082 \\
\hline$l_{9}$ & 0.105 & 0.099 & 0.072 \\
\hline$l_{10}$ & 0.119 & 0.108 & 0.085 \\
\hline$l_{11}$ & 0.113 & 0.104 & 0.079 \\
\hline$l_{12}$ & 0.129 & 0.121 & 0.093 \\
\hline$l_{13}$ & 0.104 & 0.101 & 0.074 \\
\hline$l_{14}$ & 0.109 & 0.102 & 0.076 \\
\hline$l_{15}$ & 0.105 & 0.101 & 0.075 \\
\hline$l_{16}$ & 0.182 & 0.165 & 0.123 \\
\hline
\end{tabular}

Table 7. Feature distributions (shares of feature states) for a test set (318 real estate properties)

\begin{tabular}{llllll}
\hline Feature state & Surface area & Utilities & $\begin{array}{l}\text { Transport avail- } \\
\text { ability }\end{array}$ & Neighborhood & $\begin{array}{l}\text { Physical } \\
\text { proper- } \\
\text { ties }\end{array}$ \\
\hline 0 & 0.164 & 0.000 & 0.009 & 0.016 & 0.063 \\
1 & 0.189 & 0.000 & 0.261 & 0.031 & 0.324 \\
2 & 0.648 & 1.000 & 0.730 & 0.761 & 0.613 \\
3 & $-{ }^{d}$ & - & - & 0.192 & - \\
\hline
\end{tabular}

${ }^{\mathrm{d}}$ State not present for a given feature

In ridge regression, the PE minimum was negative and closest to zero, which is the best result (due to overestimation). With regard to the first decile and third quartile, the Bayesian and mixed models were the best. For ridge regression and LASSO, 
Table 8. Percentage error (PE) characteristics

\begin{tabular}{lllllll}
\hline MPE & OLS & ME & BE & IRLS & RR & LASSO \\
\hline Min & -0.158 & -0.175 & -0.114 & -0.183 & -0.078 & 0.007 \\
First decile & -0.105 & -0.098 & -0.063 & -0.142 & 0.021 & 0.072 \\
First quartile & -0.068 & -0.066 & -0.029 & -0.110 & 0.056 & 0.089 \\
Median & 0.001 & 0.000 & 0.022 & -0.058 & 0.109 & 0.126 \\
Mean & -0.001 & -0.004 & 0.028 & -0.049 & 0.108 & 0.140 \\
Third quartile & 0.055 & 0.050 & 0.079 & 0.005 & 0.158 & 0.192 \\
Ninth decile & 0.097 & 0.095 & 0.110 & 0.052 & 0.195 & 0.222 \\
Max & 0.227 & 0.202 & 0.219 & 0.208 & 0.275 & 0.324 \\
\hline
\end{tabular}

Table 9. Absolute percentage error (APE) characteristics

\begin{tabular}{lllllll}
\hline MAPE & OLS & ME & BE & IRLS & RR & LASSO \\
\hline Min & 0.000 & 0.000 & 0.001 & 0.000 & 0.003 & 0.007 \\
First decile & 0.009 & 0.010 & 0.014 & 0.012 & 0.024 & 0.072 \\
First quartile & 0.027 & 0.026 & 0.028 & 0.030 & 0.056 & 0.089 \\
Median & 0.056 & 0.056 & 0.056 & 0.076 & 0.109 & 0.126 \\
Mean & 0.066 & 0.064 & 0.062 & 0.076 & 0.110 & 0.140 \\
Third quartile & 0.096 & 0.089 & 0.083 & 0.112 & 0.158 & 0.192 \\
Ninth decile & 0.139 & 0.127 & 0.110 & 0.143 & 0.195 & 0.222 \\
Max & 0.227 & 0.202 & 0.219 & 0.208 & 0.275 & 0.324 \\
\hline
\end{tabular}

these statistics were even positive; hence, these models gave biased (underestimated) predictions.

If the mean PE is close to zero, valuations are unbiased (regarding individual valuations). The mean PE was very close to zero for the OLS, mixed and Bayesian models, and it was very high for ridge regression and LASSO. Conclusions are similar due to the PE median. The OLS, mixed and Bayesian models perform best from the point of view of biasedness.

Analogously, if the third quartile, ninth decile and maximum of PE are smaller (positive and closer to zero), predictions are better (due to underestimation). With regard to these characteristics, IRLS and mixed estimation are the best. Furthermore, ridge regression and LASSO gave the poorest results.

For APE the lowest values are preferred. Generally, the mixed and Bayesian models are the best. Additionally, the OLS model works quite well. The first decile was the lowest for the OLS, first quartile - for mixed estimation. The APE median and mean were the best for the Bayesian, mixed and OLS models. The third and ninth deciles were the lowest for the Bayesian model, and the maximum was the lowest for mixed estimation. Generally, regarding APE, the best performing three models are the Bayesian, mixed and OLS models. The worst results are for ridge regression and LASSO.

According to the mean APE (MAPE), Bayesian and mixed models provided better predictions than OLS models. The MAPE for the mixed model was $6.4 \%$, and 
that for the Bayesian model was $6.2 \%$. For OLS it was $6.6 \%$. It is true that predictions for the mixed and Bayesian models were only slightly better, but these models (especially the mixed model) had estimates consistent with theoretical expectations. Model coefficients had proper signs, and the impact of the features was monotonic (better feature states had a greater impact). This might prove the superiority of models with prior knowledge. OLS with ridge regression and LASSO were unacceptable for practical purposes. In the case of IRLS, the MAPE was 7.6\%; hence, the predictions were worse than those for OLS, but the model coefficients also had correct signs and magnitudes, which is important if the models are to be applied by practitioners. If the OLS model is applied in an appraisal, real estate with worse feature states could have a higher value (for the OLS models, some coefficients were negative), which was unacceptable from a practical point of view.

\section{Conclusion}

Currently, information about real estate value is often needed. The number of decisions made by customers, banks, public institutions, and investors makes mass appraisal methods necessary. Individual valuations might be too time-consuming and costly. Methods applied for mass appraisals should consider the dataset quality for considered markets. For high-quality, massive dataset machine learning methods might be applied, but properly specified econometric models might also be used. However, it is different for low-quality databases from small, undeveloped and inefficient markets. For those kinds of dataset methods considering expert prior knowledge might be useful.

In the article, six econometric models were applied in real estate mass appraisal. In three of the models (mixed, Bayesian and IRLS), prior knowledge was used. This information was provided by real estate experts. Additionally, the OLS, ridge regression and LASSO models were estimated. It turned out that models with prior knowledge are better in terms of parameter efficiency and prediction accuracy. The reason for this is that databases from local real estate markets are often of low quality. Feature variability is frequently low because of the similarity of real estate. Explanatory variables are usually qualitative; hence, they are prone to subjectivity. If information quality is unsatisfactory, even sophisticated methods are useless. As a result, expert prior knowledge might in some cases improve estimation results.

Beyond the estimated models, three behaved very promisingly: the mixed, Bayesian and IRLS models. In the IRLS model, prior knowledge is rather very general, and it takes the form of restrictions in the form of inequalities. In the Bayesian approach, prior knowledge should be applied to all model parameters, which might sometimes be difficult. Therefore, the mixed model seemed to perform the best. All estimates were positive, and the impact of real estate features was monotonic. In mixed estimation, prior knowledge takes the form of stochastic restrictions imposed only on the parameters that we want. Moreover, it is important if prior information is fragmentary.

Sometimes it is stated that there is no theoretical background for applying prior knowledge. There are no strict rules here. In the article, it is suggested that prior 
knowledge might take the form of intervals for parameters. Finally, different possibilities of applying priors will be verified in future research.

Acknowledgments Project financed under the program of the Minister of Science and Higher Education under the name "Regional Initiative of Excellence" in the years 2019-2022, project number 001/ RID/2018/19, financing amount 10,684,000.00

Open Access This article is licensed under a Creative Commons Attribution 4.0 International License, which permits use, sharing, adaptation, distribution and reproduction in any medium or format, as long as you give appropriate credit to the original author(s) and the source, provide a link to the Creative Commons licence, and indicate if changes were made. The images or other third party material in this article are included in the article's Creative Commons licence, unless indicated otherwise in a credit line to the material. If material is not included in the article's Creative Commons licence and your intended use is not permitted by statutory regulation or exceeds the permitted use, you will need to obtain permission directly from the copyright holder. To view a copy of this licence, visit http://creativecommons.org/licen ses/by/4.0/.

\section{References}

Anderson, J. E. (1981). Ridge Estimation of House Value Determinants. Journal of Urban Economics, 9 , 286-297.

Benjamin, J. D., Randall, S., Guttery, R. S., \& Sirmans, C. F. (2004). Mass Appraisal: An Introduction to Multiple Regression Analysis for Real Estate Valuation. Journal of Real Estate Practice and Education, 7(1), 65-77.

Bourassa, S.C., Cantoni, E. \& Hoesli, M. (2009). Predicting House Prices with Spatial Dependence: A Comparison of Alternative Methods. Working paper presented at 15th Conference of the Pacific Rim Real Estate Society (PRRES). Sydney.

d'Amato, M. (2017). A Brief Outline of AVM Models and Standards Evolutions. In M. d'Amato \& T. Kauko (Eds.), Advances in Automated Valuation Modelling (pp. 3-21). Springer International Publishing.

Del Giudice, V., De Paola, P., Forte, F., \& Manganelli, B. (2017). Real Estate Appraisals with Bayesian Approach and Markov Chain Hybrid Monte Carlo Method: An Application to a Central Urban Area of Naples. Sustainability, 9(11), 2138. https://doi.org/10.3390/su9112138

Dell, G. (2017). Regression, Critical Thinking, and the Valuation Problem Today. Appraisal Journal, $85(3), 217-230$.

Doszyń, M. (2020). Algorithm of real estate mass appraisal with inequality restricted least squares (IRLS) estimation. Journal of European Real Estate Research, 13(2), 161-179. https://doi.org/10. 1108/JERER-11-2019-0040

Doszyń, M. (2020a). Econometric support of mass valuation process. Folia Oeconomica Stetinensia, 20(1), 81-94. https://doi.org/10.2478/foli-2020-0005

Doszyń, M. (2021). Prior information in econometric real estate appraisal: a mixed estimation procedure. Journal of European Real Estate Research, 14(3), 349-361. https://doi.org/10.1108/ JERER-11-2020-0057

Durbin, J. (1953). A Note on Regression When There is Extraneous Information About One of the Coefficients. Journal of the American Statistical Association, 48(264), 799-808. https://doi.org/10.1080/ 01621459.1953.10501201

Fik, T. J., Ling, D. C., \& Mulligan, G. F. (2003). Modelling Spatial Variation in Housing Prices: A Variable Interaction Approach. Real Estate Economics, 31(4), 623-646.

Gnat, S., \& Doszyń, M. (2020). Parametric and Non-parametric Methods in Mass Appraisal on Poorly Developed Real Estate Markets. European Research Studies Journal, 23(4), 1230-1245. https://doi. org/10.35808/ersj/1740

Grömping, U. (2010). Inference with Linear Equality and Inequality Constraints Using R: The Package ic.infer. Journal of Statistical Software, 33(10), 1-31. 
Isakson, H. R. (1998). The Review of Real Estate Appraisals Using Multiple Regression Analysis. Journal of Real Estate Research, 15(2), 177-190.

Jahanshiri, E., Buyong, T., \& Shariff, A. R. M. (2011). A Review of Property Mass Valuation Models. Pertanika Journal of Science \& Technology, 19, 23-30.

James, G., Witten, D., Hastie, T. \& Tibshirani R. (2021). An Introduction to Statistical Learning with Applications in $R$. Second Edition. Springer Texts in Statistics.

Judge, G. G., \& Takayama, T. (1966). Inequality Restrictions in Regression Analysis. Journal of the American Statistical Association, 61(313), 166-181. https://doi.org/10.1080/01621459.1966.10502 016

Kauko, T., \& d'Amato, M. (Eds.). (2008). Mass Appraisal Methods: An International Perspective for Property Valuers. Blackwell Publishing Ltd..

Kilpatrick, J. (2011). Expert Systems and Mass Appraisal. Journal of Property Investment \& Finance, 29(4/5), 529-550.

Koop, G. (2003). Bayesian econometrics. John Wiley \& Sons.

Liew, C. K. (1976). Inequality Constrained Least-Squares Estimation. Journal of the American Statistical Association, 71(355), 746-751.

Lovell, M. C., \& Prescott, E. (1970). Multiple Regression with Inequality Constraints: Pretesting Bias, Hypothesis testing and Efficiency. Journal of the American Statistical Association, 65(330), 913-925.

McCluskey, W. J., McCord, M., Davis, P. T., Haran, M., \& Mcllhatton, D. (2013). Prediction Accuracy in Mass Appraisal: a Comparison of Modern Approaches. Journal of Property Research, 30(4), 239-265.

Mehta, J. S. \& Swamy, P. A. V. B (1970). The Finite Sample Distribution of Theil's Mixed Regression Estimator and a Related Problem. Review of the International Statistical Institute, 38(2). https:// www.jstor.org/stable/1402143?seq=1\&cid=pdf-

Mittelhammer, R. C., \& Conway, R. K. (1988). Applying Mixed Estimation in Econometric Research. American Journal of Agricultural Economics, 70(4), 859-866.

Moore, J. S., Reichert, A. K., \& Cho, C. (1984). Analyzing the Temporal Stability of Appraisal Model Coefficients: An Application of Ridge Regression Techniques. AREUEA, 12, 50-71.

Nagar, A. L., \& Kakwani, N. C. (1964). The Bias and Moment Matrix of a Mixed Regression Estimator. Econometrica, 32(1/2), 174-182. https://doi.org/10.2307/1913742

Newell, G. J. (1982). The application of ridge regression to real estate appraisal. Appraisal Journal, 50(1), 116-119.

Pace, R. K. (1995). Parametric, semiparametric, and nonparametric estimation of characteristic values within mass assessment and hedonic pricing models. The Journal of Real Estate Finance and Economics, 11, 195-217. https://doi.org/10.1007/BF01099108

Pace, R. K., \& Gilley, O. W. (1990). Estimation employing a priori information within mass appraisal and hedonic pricing models. The Journal of Real Estate Finance and Economics, 3(1), 55-72. https:// doi.org/10.1007/BF00153706

Pagourtzi, E., Assimakopoulos, V., Hatzichristos, T., \& French, N. (2003). Real Estate Appraisal: a Review of Valuation Methods. Journal of Property Investment \& Finance, 21(4), 383-401.

Sing, T. F., Yang, J. J., \& Yu, S. M. (2021). Boosted Tree Ensembles for Artificial Intelligence Based Automated Valuation Models (AI-AVM). Journal of Real Estate Finance and Economics. https:// doi.org/10.1007/s11146-021-09861-1

Swamy, P. A. V. B., \& Mehta, J. S. (1969). On Theil's Mixed Regression Estimator. Journal of the American Statistical Association, 64(325), 273-276.

Theil, H. (1963). On the Use of Incomplete Prior Information in Regression Analysis. Journal of the American Statistical Association, 58(302), 401-414.

Theil, H., \& Goldberger, A. S. (1961). On Pure and Mixed Statistical Estimation in Economics. International Economic Review, 2(1), 65-78. https://doi.org/10.2307/2525589

Toker, S., Şiray, G. U., \& Kaçıranlar, S. (2013). Inequality Constrained Ridge Regression Estimator. Statistics and Probability Letters, 83, 2391-2398.

Wang, D., \& Li, V. J. (2019). Mass Appraisal Models of Real Estate in the 21st Century: A Systematic Literature Review. Sustainability, 11(24), 7006. https://doi.org/10.3390/su11247006

Werner, H. J. (1990). On Inequality Constrained Generalized Least-Squares Estimation. Linear Algebra and Its Applications, 127, 379-392.

Wolak, F. A. (1989). Testing inequality constraints in linear econometric models. Journal of Econometrics, 41(2), 205-235. https://doi.org/10.1016/0304-4076(89)90094-8 
Zellner, A. (1961). Linear Regression with Inequality Constraints on the Coefficients: an Application of Quadratic Programming and Linear Decision Rules. International Centre for Management Science. Report 6109 (MS No. 9)

Zurada, J., Levitan, A. S., \& Guan, J. (2011). A Comparison of Regression and Artificial Intelligence Methods in a Mass Appraisal Context. Journal of Real Estate Research, 3, 349-387.

Publisher's note Springer Nature remains neutral with regard to jurisdictional claims in published maps and institutional affiliations. 